\title{
Study on Dynamic Reliability of Barrel Shell Based on RBF Neural Network SONG Zhi-fei ${ }^{1}$, LI Hui-jun ${ }^{2}$, Li Biao ${ }^{1}$, Qin Jin-lei ${ }^{1}$ \\ 1. College of Architecture and Civil Engineering, North China University of Technology, Beijing 100041, China \\ 2. College of Water Resources and Architectural Engineering, Northwest A\&F University, Yangling 712100, China
}

Keywords: Dynamic reliability; RBF neural network; Response Surface Method; First Excursion Failure Criterion; Linearized Nataf transformation

\begin{abstract}
An algorithm for dynamic reliability analysis of a stochastic structure is proposed based on the theory of Neural Network Response Surface Method. On the basis of First Excursion Failure Criterion of random vibration, the performance function of dynamic reliability is established. Sequential Surface Response Method is introduced, and subsequently three-layer BP network is used to fit the performance function. A new method combining linearized Nataf transformation and iHLRF algorithm is proposed, which is introduced to compute dynamic reliability in barrel shell. Compared with other existing methods, the proposed method not only markedly reduces the iterations times, but also have better accuracy. It has wide prospect in dynamic reliability of spatial structures.
\end{abstract}

\section{Introduction}

For the complexity of large-span lattice structures, all kinds of undetermined factors are needed to take into consideration. Explicit solutions are uneasy to be obtained analytically duo to performance function is highly nonlinear. Monta-Carlo Method is precise but time-consuming. The concept of stochastic finite element is clear but it is uneasy to program, furthermore, great error may be generated for nonlinear problem and/or large variation coefficients of random variables. Fortunately, Response Surface Method has been widely applied ${ }^{[1]}$. On the basis of First Excursion Failure Criterion of random vibration, the performance function of dynamic reliability is established. Sequential Surface Response Method is introduced, and subsequently three-layer BP network is used to fit the performance function. A new method combining linearized Nataf transformation and iHLRF algorithm is proposed.

\section{Presentation of New Algorithm for Dynamic Reliability}

\subsection{Performance function}

Response of structure under stochastic loads is a random process and changing with time, hence performance function is described as follows ${ }^{[2]}$ :

$$
\mathrm{z}_{\min }(\mathrm{t})=\mathrm{L}-\mathrm{y}_{\mathrm{max}}(t)
$$

\footnotetext{
${ }^{1}$ SONG Zhi-fei (1980-), Ph.D, Associate Professor, E-mail: song59672@163.com
} 
where $\underset{\substack{\max \\ \mathrm{t}[0, \mathrm{~T}]}}{ }(t)$ is the maximal value of structural response. It is obtained based on First Excursion Failure Criterion of random vibration, for a linear structure it can be expressed as follows:

$$
\mathrm{y}_{\max }(t)=\eta \sigma_{y}
$$

The mean, variance and distribution function of $\eta$ are as follows:

$$
\eta=\left(2 \ln N_{0}^{+} T\right)^{\frac{1}{2}}+\frac{0.5772}{\left(2 \ln N_{0}^{+} T\right)^{\frac{1}{2}}}, \quad \sigma_{\eta}^{2}=\frac{\pi^{2}}{6}\left(2 \ln N_{0}^{+} T\right)^{-1}, F(\eta)=\exp \left(-N_{0}^{+} T \exp \left(-\frac{\eta^{2}}{2}\right)\right)
$$

$\sigma_{\mathrm{y}}$ is response total root mean square, and root mean square of single-freedom system can be obtained according to following equation:

$$
\sigma_{y}=\sqrt{\left.\int_{-\infty}^{+\infty} H(\omega)\right|^{2 n} s(\omega) d \omega}
$$

where $H(\omega)$ is frequency response function of system. For multi-degree of freedom system, $\sigma_{y}$ is fitted by Sequence Response Surface Method.

\subsection{Theory and algorithm of neural network response surface method}

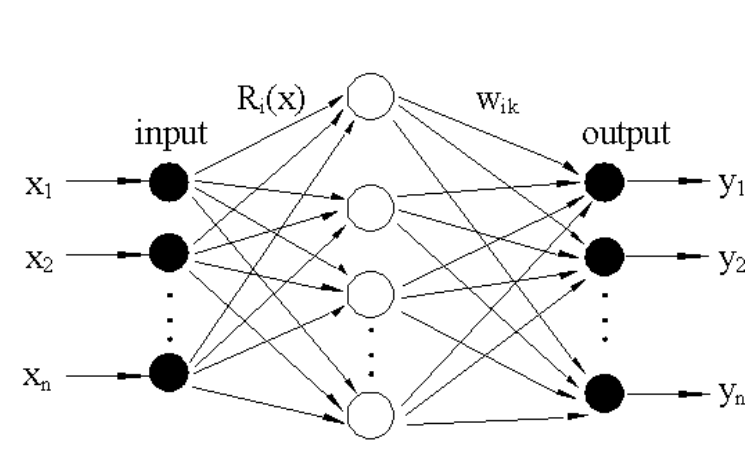

Fig.1 RBF neural network

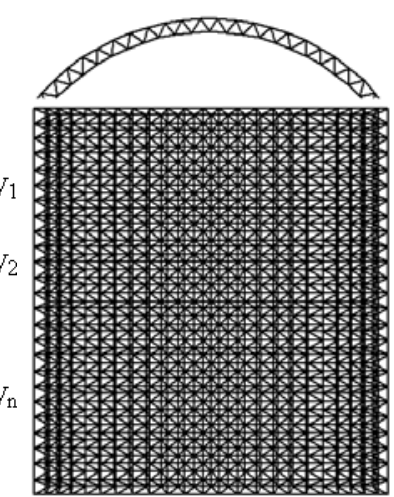

Fig. 2 double-layer cylindrical latticed shell

As we all know, precision of Response Surface Method can be solved theoretically by BP network, but BP network has low convergence speed and local minimum problem ${ }^{[3]}$. Radial base function network ${ }^{[4]}$ has better analytical ability and higher learning speed than BP network. Radial Basis Function, RBF, consists of three layers, and its structure is shown in Fig.1. Gauss function is usually applied due to its simple form, good smoothness and arbitrary-order derivability. Its expression is shown as follows ${ }^{[5]}$ :

$$
R_{i}(x)=\exp \left[-\frac{\left\|x-c_{i}\right\|^{2}}{2 \sigma_{i}^{2}}\right], i=1,2, \cdots, m
$$

where $x$ is $n$-dimensional vector; $c_{i}$ is the center of $i$ th base function. $\sigma_{\mathrm{i}}$ is a perceptive variable $i$ th, $\left\|x-c_{i}\right\|$ is the norm of $x-c_{i}$. From Fig. 1 we can see that nonlinearity mapping of $x \rightarrow R_{i}(x)$ has been implemented ${ }^{[6]}$, and fitting expression is obtained as follows:

$$
y_{k}=\sum_{i=1}^{m} \omega_{i k} R_{i}(x)=\sum_{i=1}^{m} \omega_{i k} \exp \left[-\frac{\left\|x-c_{i}\right\|^{2}}{2 \sigma_{i}^{2}}\right], k=1,2, \cdots, p
$$


Fitting expression of performance function of stochastic structural dynamic reliability is obtained as follows:

$$
Z^{\prime}=L-\eta \sigma_{y}^{\prime}=L-\eta \sum_{i=1}^{m} \omega_{i k} \exp \left[-\frac{\left\|X-c_{i}\right\|^{2}}{2 \sigma_{i}^{2}}\right]
$$

Finally, $\sigma_{\mathrm{y}}$ is obtained by FEM analysis. Error diffusion is used to learning samples. Subsequently, dynamic reliability index can be obtained by combining linearized Nataf transformation and iHLRF algorithm.

\subsection{Calculation procedures}

The calculation procedures in dynamic reliability analysis as follows: 1) Mean values of random variables have been applied, hence positive slope expectation crossing-zero rates can be obtained by finite element analysis, finally, mean and variance of $\eta$ can been obtained, 2) taking mean value of random variables as central point of samples, 3) choosing $2 n+1$ sample sets, and response total root mean square $\sigma_{\mathrm{y}}$ can be obtained by finite element analysis. 4)choose sampling points as neural network input data, and compare $\sigma_{\mathrm{y}}$ obtained by network mapping to one obtained by FEM analysis, finally calculate network error, 5) estimate whether the error satisfy the prescribed precision or not, if does go to 7); if doesn't, inversely distribute error to each element, and adjust threshold of connecting weight, 6) return to 4), learn recurrently, until network error satisfy tolerable error, 7) fit function based on determined neural network mapping relations, 8) dynamic reliability index $\beta$ can be obtained by combination of linearized Nataf and iHLRF algorithm.

\section{Dynamic reliability analysis of barrel shell}

\subsection{Model description}

Study on barrel shell with span $80 \mathrm{~m}$. Longitudinal dimension is $87.5 \mathrm{~m}$, curvature height is $20 \mathrm{~m}$. Member section is $\Phi 121 \times 4.0$. Shell is excited by displacement excitation spectrum in Y direction, and values are tabulated in Table 1. Plane and elevation of barrel shell are shown in Figure 2.

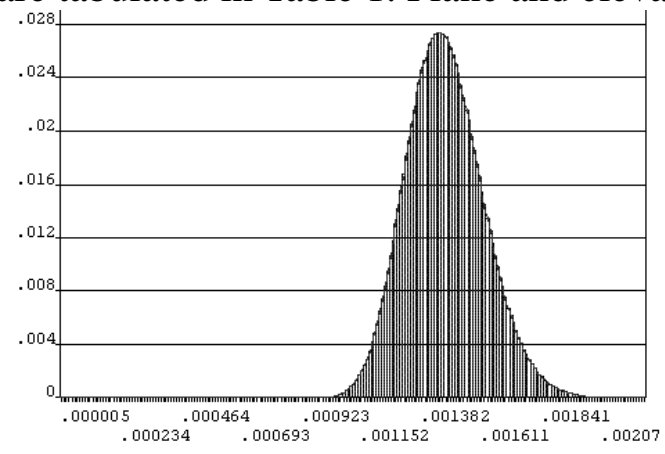

Fig. 3 histogram figure of sectional-area of bars

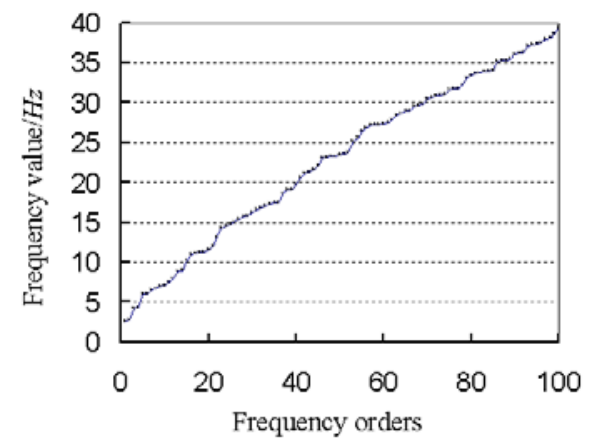

Fig. 4 curve of first 100 frequency of shell

Tab.1 Displacement excited spectrum

\begin{tabular}{|c|c|c|c|c|c|c|c|c|}
\hline Frequency, $\mathrm{Hz}$ & 0.5 & 1.0 & 2.4 & 3.8 & 17 & 18 & 20 & 32 \\
\hline Displacement, $\mathrm{mm}$ & 0.01 & 0.016 & 0.03 & 0.02 & 0.005 & 0.01 & 0.015 & 0.01 \\
\hline
\end{tabular}

\subsection{Probabilistic model of loads, elastic modulus and section dimension of member}

Random variables of dead load, elastic modulus, member cross-sectional area are tabulated as Table 2. Histogram of cross-sectional area is shown in Figure 3. 
Tab.2 Statistical parameters of random variables

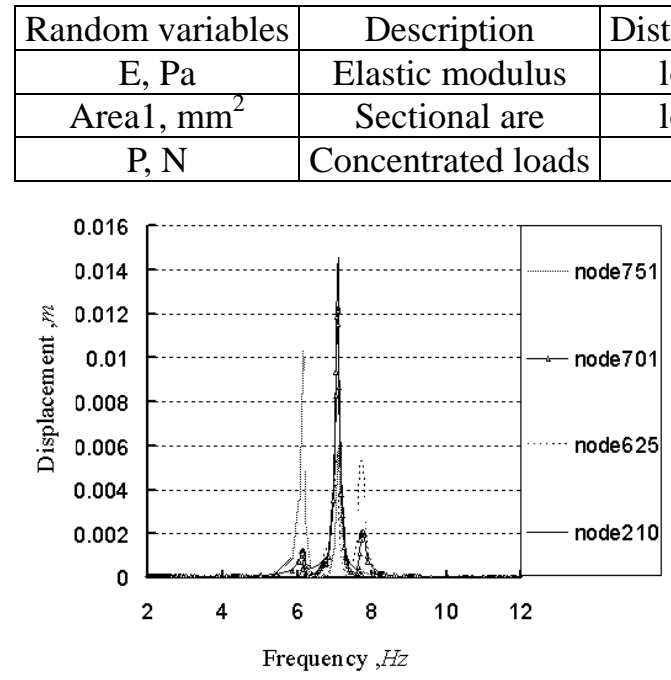

Fig.5 a frequency range is $2 \sim 12 \mathrm{~Hz}$

\begin{tabular}{|c|c|c|}
\hline Distribution type & Mean & Standard deviation \\
\hline lognormal & $2.1 \times 10^{11}$ & $4.2 \times 10^{9}$ \\
lognormal & 1470.27 & 147.027 \\
\hline normal & 24659.84 & 1721.536 \\
\hline
\end{tabular}

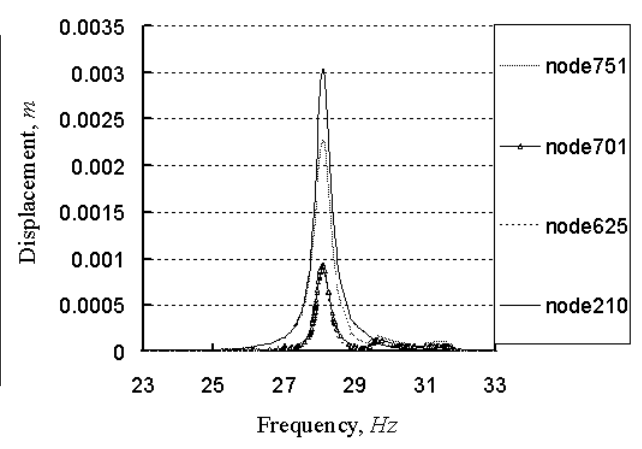

Fig. 5 b frequency range is $23 \sim 33 \mathrm{~Hz}$

Fig. 5 power spectrum response of displacement of nodes

\subsection{Dynamic reliability analysis of double-layer barrel lattice shell}

Considering the complexity of shell and prevent eigenvalue from missing, the first 100 frequencies are considered in the modal analysis, the first 100 frequencies are shown in Figure 4. Results show that frequencies are dense, and the first frequency is $2.5376 \mathrm{~Hz}$, and the hundredth frequency is $39.463 \mathrm{~Hz}$.

Computation follows the procedure in section 2.4, and 13-time finite element dynamic response is performed. Based on OpenSees, combination between linearized Nataf transformation and iHLRF algorithm is implemented, and $\beta$ is obtained after 12-time iterations. Displacement power response spectrum curves of node 751,701,625 and 210 of shell are shown in Figure 5, displacement power response spectrum curves corresponding to frequency range $2 \sim 12 \mathrm{~Hz}$ and $23 \sim$ $33 \mathrm{~Hz}$ are shown in Figures $5 \mathrm{a}$ and $5 \mathrm{~b}$, respectively; sensitivity diagram of response total mean square root to sectional area, loads and elastic modulus are shown in Figure 6, from which we can see that response total mean square root is mainly affect by sectional area of member, and elastic modulus and loads slightly influence the value; response surface of $\sigma_{\mathrm{y}}$ to elastic modulus and of sectional area of member is shown in Figure 7.
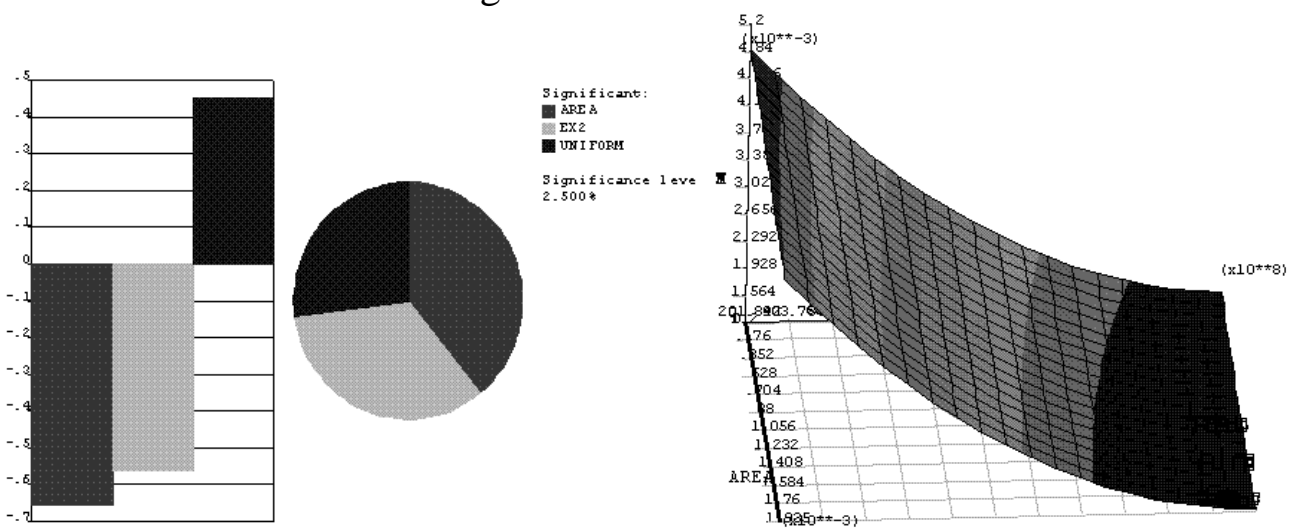

Fig.6 sensitivity diagram of $\sigma_{y}$ to area, loads and $\mathrm{E}$ Fig. 7 area-modulus- $\sigma_{y}$ response surface

To verify the precision and efficiency of the method, Monta-Carlo method has been presented. 10000 samples are extracted by ANSYS. The results are shown in Table 3. From that we can see the method has excellent computation accuracy and efficiency. 
Tab.3 Comparison of results between two methods

\begin{tabular}{|c|c|c|c|c|}
\hline Computed method & Calculation times, $\mathrm{n}$ & Time, $\mathrm{h}$ & Reliability index, $\beta$ & Error \\
\hline Method in the study & 13 & 0.6 & 3.512 & \multirow{2}{*}{$0.94 \%$} \\
\hline Monta-Carlo Method & 10000 & 34 & 3.545 & \\
\hline
\end{tabular}

\section{Conclusions}

This paper proposes a new algorithm to compute dynamic reliability of large-span spatial latticed structures, and the method base on Neural Network Response Surface Method. Based on First Excursion Failure Criterion of random vibration, the performance function of dynamic reliability is established according to performance function mode of random variables. Sequential Surface Response Method is introduced, and subsequently three-layer BP network is applied to fit the performance function. Neural network has strong flexibility and can generate arbitrary shape. It is more flexible to traditional polynomial function. A new reliability method combining linearized Nataf transformation and iHLRF algorithm is presented. It is the first time to introduce the proposed algorithm to compute dynamic reliability in long-span spatial reticulated steel structures.

\section{References}

[1] HUANG Liang, YI Wei-jian, WANG You. Improvement study on response surface method for reliability analysis in geotechnical engineering [J]. Rock and Soil Mechanics, 2008,39(2):370-374.

[2] Chen ying, Wang Dongsheng, Zhu Changchun. Dynamic reliability analysis of stochastic structures subjected to random loads [J]. Engineering Mechanics, 2006,23(10): 82-85.

[3] WEN Xin, ZHOU Lu, WANG Dan-li, etc. Neural network design for application with Matlab [M]. Beijing: Science Press, 2000:244-248.

[4] Bucher C G, Bourgund U. A fast and efficient response surface approach for structural reliability problems [J]. Structural Safety, 1990,7(1):57 66.

[5] Liu P-L, Der Kiureghian A. Multivariate distribution models with prescribed marginal and covariance [J]. Probabilistic Engineering Mechanics, 1986, 1(2): 105 112.

[6] LIU Chunguang, Li Huijun. Effect of optimization based on geometrical parameters on dynamic performance of double-layer barrel shell [J]. Journal of Disaster Prevention and Mitigation Engineering, 2008,28(3): 342-348. 